\title{
Philonsorbonne
}

6 | 2012

Année 201 1-2012

\section{Temps de la guerre civile, temps de l'intervention, selon John Stuart Mill}

\section{Aurélie KNÜFER}

\section{Q OpenEdition}

\section{Journals}

Édition électronique

URL : https://journals.openedition.org/philonsorbonne/380

DOI : 10.4000/philonsorbonne.380

ISSN : $2270-7336$

Éditeur

Publications de la Sorbonne

\section{Édition imprimée}

Date de publication : 15 juillet 2012

Pagination : 23-39

ISBN : 978-2-85944-710-6

ISSN : 1255-183X

\section{Référence électronique}

Aurélie KNÜFER, «Temps de la guerre civile, temps de l'intervention, selon John Stuart Mill »,

Philonsorbonne [En ligne], 6 | 2012, mis en ligne le 04 février 2013, consulté le 08 juin 2021. URL

http://journals.openedition.org/philonsorbonne/380; DOI : https://doi.org/10.4000/philonsorbonne. 380

(c) Tous droits réservés 


\title{
Temps de la guerre civile, temps de l'intervention, selon John Stuart Mill
}

\author{
Aurélie Knüfer
}

On trouverait difficilement une théorie de la guerre civile chez John Stuart Mill : les nombreux articles qu'il consacre aux conflits intra-étatiques, aux guerres de libération et aux insurrections de son temps ne visent pas à découvrir sous la multiplicité des conflits, une essence de la guerre civile, mais ils doivent plutôt se lire, semble-t-il, comme des analyses circonstanciées de luttes singulières, ayant chacune ses enjeux, ses protagonistes et ses traits propres ${ }^{1}$. Spectateur parfois enthousiaste, parfois critique de ces conflits, il n'entend donc pas les penser en rapport avec l'essence de la politique, ou avec celle des guerres interétatiques et il souhaite les juger en toute impartialité ${ }^{2}$.

1. Il n'est pas possible d'énumérer ici l'ensemble des textes dans lesquels Mill évoque les conflits et les guerres civiles de son temps, tant les occurrences de cette question sont nombreuses dans son œuvre, en particulier dans ses articles et dans ses lettres. L'entrée de l'expression civil war, dans l'index des œuvres complètes permet de mesurer l'importance de ce problème (John Stuart Mill, Indexes, Collected Works, London, Routledge, 1991, vol. XXXIII, p. 503). Remarquons cependant que ce que nous entendrons ici par "guerre civile » reçoit chez Mill plusieurs noms. Il emploie en effet indistinctement, pour désigner les conflits intra-étatiques, les termes suivants : civil war, contest, insurrection et rebellion. Il n'existe pas de définition en bonne et due forme de la guerre civile chez Mill, ou d'interrogation sur les différentes modalités d'affrontement ou de déchirement d'un peuple. Nous entendrons donc par «guerre civile », d'une manière très générale, toute lutte armée mettant aux prises plusieurs fractions d'un État ou d'une nation.

2. Dans son livre intitulé : «The Contest in America », Mill écrit : «Je n'ai pas peur du mot rébellion. Je n'ai aucun scrupule à dire que j'ai sympathisé plus ou moins ardemment avec la plupart des rébellions, victorieuses ou non, qui ont eu lieu à mon époque ». Il rajoute cependant: «Mais je n'ai pour autant jamais pensé que le simple fait de se rebeller constituait un titre suffisant pour recevoir ma sympathie, ou que le fait de prendre les armes contre ses concitoyens était méritoire en lui-même [...]. Il me semble que la doctrine selon laquelle l'action humaine la plus sérieuse et la plus responsable n'obligerait pas ceux qui 
Il est cependant possible de trouver, entre ces analyses, un dénominateur commun. En effet, il s'agit presque à chaque fois pour Mill d'étudier les guerres civiles non pas pour elles-mêmes, comme si elles pouvaient être soustraites à l'ordre du monde et que leur issue n'engageait que l'avenir de leur État ou de leur nation, mais plutôt comme étant déjà prises dans une totalité, et comme concernant au plus haut point l'ensemble des autres peuples. Il faut même aller plus loin et dire que si les guerres civiles sont analysées c'est, d'une part, à travers le prisme de leur réception, de la manière dont elles sont perçues et dont elles agissent sur les autres peuples, et d'autre part, sous l'horizon de la question de savoir comment des États, ou des peuples déjà libres, peuvent ou doivent se comporter face à des peuples qui se libèrent du joug de leur tyran. Autrement dit, les guerres civiles, chez Mill, engagent d'emblée les autres États et les autres peuples en ce qu'elles les obligent à penser aux conflits qui adviennent hors de leurs frontières, à les juger sur le plan moral, mais aussi à se demander ce que les guerres civiles exigent d'eux, sur le plan pratique, politique, et militaire.

C'est donc sous cet angle que nous voudrions interroger trois textes de John Stuart Mill : "Vindication of the French Revolution of February $1848 »(\text { datant de } 1849)^{3}$, «Few Words on Non-Intervention $(1859)^{4}$, et «The Contest in America» $(1862)^{5}$. Si ces trois textes présentent les traits communs que nous venons de signaler, ils constituent cependant trois modèles distincts du rapport qu'un peuple libre, ou qu'un gouvernement libre, devrait entretenir, pour Mill, avec un peuple qui se libère dans une guerre civile. Bien qu'on retienne le plus souvent de John Stuart Mill son texte fameux de 1859, qui vise à fonder en raison la non-intervention ${ }^{6}$, l'étude de ces textes pourra donner à voir les ambiguïtés, voire la réversibilité de son opposition à l'intervention militaire. Or, ce que nous voudrions montrer ici, c'est que les réponses distinctes, ou contradictoires,

l'accomplissent à montrer qu'ils ont un véritable motif de plainte, est bien étrange [...]». (Essays on Equality, Law and Education, CW, vol. XXI, p. 137).

3. J. S. Mill, «Vindication of the French Revolution of February 1848 », in Essays on French History and Historians, CW, vol. XX, p. 319-363.

4. J. S. Mill, «A Few Words on Non-Intervention», in Essays on Equality, Law, and Education, CW, vol. XXI, p. 111-124.

5. J. S. Mill, «The Contest in America », in CW, vol. XXI, p. 127-142.

6. C'est Michael Walzer qui, semble-t-il, a d'abord redonné une actualité à ce texte de Mill (M. Walzer, Guerres justes et injustes, trad. S. Chambon et A. Wicke, Paris, Gallimard, 2006 [1975], p. 183-219). Après lui, on peut penser à l'ouvrage de Charles R. Beitz, Political theory and International relations, Princeton, Princeton University Press, 1999 [1979], p. 83-115. Plus récemment, Micheal Doyle a examiné dans un article le principe de non-intervention et l'intervention à partir du texte de Mill (M. Doyle, "The New Interventionism », Metaphilosophy, Vol. 32, Janvier 2011, p. 214-221. Enfin, Arthur Isak Applbaum, a analysé la question de savoir s'il est possible de forcer un peuple à être libre, en s'appuyant sur les analyses de Mill (A. I. Applbaum, «Forcing a People to Be Free », Philosophy and Public Affairs, 35, $\mathrm{n}^{\circ}$ 4, 2007, p. 359-400). Le point commun entre ces textes est qu'ils évoquent seulement «A Few Words on Non-Intervention », sans prendre la peine d'interroger les autres textes consacrés par Mill à cette question. 
apportées par Mill à la question de l'intervention dans une guerre civile étrangère, sont étroitement corrélées à la façon dont il pense la temporalité de la guerre civile, c'est-à-dire la manière dont la liberté s'y réalise dans le temps. Aussi, si ces trois textes apportent des réponses différentes au problème de l'intervention militaire, ce serait parce qu'ils pensent différemment la manière dont la liberté s'actualise, se crée, ou se régénère dans le temps de la guerre civile.

\section{«Vindication of the French Revolution of February 1848 » : la guerre civile comme actualisation du libéralisme}

\section{L'âge des « sympathies libérales »}

Notre premier texte, «Vindication of the French Revolution of February $1848 »$, est une défense de la Révolution de février, contre les attaques de pamphlétaires anglais. Il s'agit notamment pour Mill de défendre la politique extérieure de Lamartine, accusé par ses détracteurs d'avoir l'intention d'exporter la révolution et d'aider les peuples à renverser leurs gouvernements. L'enjeu du texte de Mill est double: il veut à la fois montrer que les intentions de Lamartine sont pacifiques et que celui-ci n'a pas pour but d'interférer dans les affaires intérieures des autres États en provoquant des guerres civiles, mais il souhaite également défendre le droit d'intervention, en montrant qu'il devrait avoir sa place dans un nouveau droit des gens, adapté à une époque nouvelle. Il écrit :

Y a-t-il une exigence [...] plus forte - y a-t-il un motif d'intervenir d'un caractère plus contraignant - que celui d'empêcher que la liberté d'une nation, qui se soucie suffisamment de la liberté pour la revendiquer en prenant les armes, soit écrasée et piétinée par ses oppresseurs tyranniques, qui n'ont ni le même nom, ni le même sang qu'elle, mais qui sont des conquérants étrangers ? Les coutumes, faussement appelées droit des gens, établies dans les livres, ont été faites pour un âge comme celui de Louis XIV, et pour empêcher les despotes puissants et ambitieux d'avaler les États plus petits. Elles étaient bien adaptées à ce but. Mais les grands intérêts des nations civilisées, aujourd'hui, ne consistent pas dans l'attaque et la défense du territoire, mais dans la liberté, la justice du gouvernement, et la sympathie de l'opinion. Ce n'est pas pour cet état de choses que le droit des gens a été fait [...]. Il y a eu autrefois en Europe un temps où, comme aujourd'hui, les intérêts les plus forts des nations, aussi bien sur le plan domestique que sur le plan des relations étrangères, étaient les intérêts de l'opinion : c'était l'ère de la Réforme. Quelqu'un prêta-t-il alors la moindre attention au principe de non-intervention ? La sympathie religieuse n'était-elle pas considérée comme une justification suffisante pour apporter son aide? Les protestants n'aidaient-ils pas les protestants, quand ils étaient menacés par leur propre gouvernement ? Les catholiques n'aidaient-ils pas les catholiques à supprimer l'hérésie ? Ce que les sympathies religieuses étaient alors, les sympathies politiques le sont à présent ; et tout gouvernement ou tout peuple libéral a le droit d'aider le libéralisme en lutte, par la médiation, par 
l'argent, par les armes, dès lors qu'il peut le faire avec prudence; de même que tout gouvernement despotique, quand on l'appelle à l'aide, n'a jamais de scrupules à aider les gouvernements despotiques .

On peut se demander ce que Mill entend exactement par «liberté », «justice du gouvernement» et «sympathie de l'opinion», qui constitueraient les nouveaux «intérêts» des États. Alors qu'à l'âge de Louis XV, c'était les questions de territoire qui intéressaient les despotes, à l'époque de l'extension de la démocratie, ou de la liberté, le souci d'un État serait d'abord de savoir s'il est entouré d'États libres ou pas. Si Mill n'explique pas ce point, on pourrait penser que pour lui, suivant en cela la thèse de Kant dans Vers la paix perpétuelle, les Républiques ou les gouvernements populaires, seraient moins enclins à faire la guerre ${ }^{8}$. Dès lors, il y aurait un intérêt pour les États à s'assurer qu'ils sont bien entourés de gouvernements justes, et de peuples libres, puisque seule une telle configuration leur garantirait la sécurité. Cependant, ce que Mill entend par «sympathie de l'opinion» n'est pas clair. Ceci signifie-t-il que les gouvernements devraient faire en sorte de défendre les peuples pour lesquels l'opinion aurait de la sympathie, ou bien qu'ils devraient protéger les peuples qui partageraient la même opinion, à savoir l'opinion libérale ? En outre, avoir de la «sympathie politique », est-ce simplement se reconnaître des points communs ou un air de famille avec certaines aspirations et luttes politiques, ou bien est-ce partager les mêmes principes, les mêmes convictions et les mêmes croyances ? Le texte ne semble pas permettre, à première vue, de lever ces ambiguïtés. Cependant, on peut remarquer qu'en affirmant la priorité de la «liberté » et de la «sympathie » sur les questions de territoire, tout se passe comme si Mill faisait éclater les frontières des États, et rendait la notion même de guerre civile problématique, en ce que tout conflit pour la liberté engagerait immédiatement les autres peuples et les autres gouvernements. Autrement dit, l'idée même d'un conflit intra-

7. J. S. Mill, op. cit., p. 346.

8. Dans le «premier article définitif pour la paix perpétuelle », Kant écrit en effet : «[...] la constitution républicaine offre, outre la pureté de son origine, puisqu'elle provient de la source pure du concept de droit, également la perspective de conduire au résultat souhaité, à savoir la paix perpétuelle ». La raison en est, selon Kant, que les citoyens, dont le consentement est exigé en République, ne déclareront jamais la guerre pour des motifs insignifiants, puisque précisément ce sont eux qui en supporteraient la charge (E. Kant, Vers la paix perpétuelle, trad. M. Marcuzzi, Paris, Vrin, 2007, p. 26). Il ne semble pas cependant que Mill ait eu connaissance de ce texte, qu'il ne cite jamais. Notons que l'idée d'un lien intrinsèque entre république et paix apparaît également chez Montesquieu. Exposant les avantages de la constitution fédérative et montrant qu'elle «doit être composée d'États de même nature », il écrit : «L'esprit de la monarchie est la guerre et l'agrandissement : l'esprit de la république est la paix et la modération. Ces deux formes de gouvernement ne peuvent, que d'une manière forcée, subsister dans une république fédérative. » (Montesquieu, De l'Esprit des lois, Paris, Garnier-Flammarion, 1979, IX, 2, p. 267) Rien ne nous permet d'affirmer, cependant, que Mill ait eu connaissance de ces lignes : les références qu'il fait à L'Esprit des lois dans son œuvre laissent penser que sa connaissance de Montesquieu est assez superficielle et peut-être seulement de seconde main. 
étatique semble caduque, puisque toute guerre civile a d'emblée une dimension internationale.

\section{«Libéralisme en lutte » et souci de la liberté}

L'expression que Mill emploie à la fin de notre texte, lorsqu'il écrit que «tout gouvernement et tout peuple libéral a le droit d'aider le libéralisme en lutte [struggling liberalism] », peut nous permettre de répondre à la question que nous avons posée plus haut au sujet de la sympathie. Parler de «libéralisme en lutte », plutôt que de «peuple luttant pour sa liberté », semble indiquer que pour Mill, les guerres civiles ne sont que le moment de l'actualisation d'un même principe politique. Autrement dit, loin de constituer des mouvements indépendants et singuliers, les guerres civiles permettraient de faire émerger sur la scène européenne ce qui y serait déjà à l'œuvre souterrainement, à savoir le libéralisme. Ainsi, nous n'aurions pas affaire, à chaque fois, à un peuple qui se bat pour assurer son autonomie, mais ce serait le «libéralisme » lui-même qui combattrait dans les guerres civiles la tyrannie.

Il faut remarquer que Mill ne propose pas ici de définition du libéralisme. On trouverait d'ailleurs difficilement une définition du libéralisme dans l'ensemble de son œuvre. On peut penser cependant que par libéralisme, il entend la doctrine politique qui défend l'égalité des droits et des conditions, c'est-à-dire la démocratie - une telle équivalence apparaît en tout cas dans l'essai qu'il consacre à Tocqueville en $1830^{9}$. Or, ce qui apparaît clairement ici, c'est que si la démocratisation ou la libéralisation peut prendre différentes formes, s'établir selon des rythmes distincts, ce serait un même principe qui serait à l'œuvre chez tous les peuples et tous les États civilisés. Ainsi, la différence entre les modalités d'actualisation de cette liberté serait contingente au regard de l'univocité de la liberté qui se réalise.

C'est d'ailleurs cette "univocité » de la liberté ou du libéralisme qui semble autoriser les gouvernements et les peuples à voir dans le simple soulèvement des insurgés le signal que le mouvement de libération ou de libéralisation est en marche. Aussi, le simple fait que le peuple «prenne les armes », comme l'écrit Mill au début de notre texte, peut être

\footnotetext{
9. Au début de l'essai, Mill montre en effet que l'impartialité de Tocqueville, à l'égard de la démocratie et de l'aristocratie, est le fruit d'une double éducation, qui lui aurait permis de connaître et d'apprécier ces deux formes de gouvernement. Il écrit en effet : «Mais entre l'aristocratie et la démocratie, il maintient la balance égale, avec cette entière absence de passion que l'on attend d'un observateur scientifique. Il était assurément très bien placé pour considérer chacune des deux positions de cette grande controverse avec un jugement impartial. Les sentiments de sa première éducation étaient royalistes, alors que le libéralisme constitue le courant prédominant dans la société de notre siècle. » (J. S. Mill, «M. de Tocqueville et la démocratie en Amérique [I] », in Essais sur Tocqueville et la société américaine, trad. P.-L. Autin, M. Garandeau, E. Marquer, E. Mignot et P. Thierry, Paris, Vrin, 1994, p. 52).
} 
immédiatement interprété par les spectateurs étrangers comme le signe d'un « souci de la liberté » [care for liberty]. Tandis que la guerre civile dans les «A Few Words on non-intervention » deviendra le moment où le peuple apprend progressivement à aimer ou à désirer la liberté, ici, le simple fait du soulèvement constituerait un signe suffisant de l'intérêt qu'un peuple lui accorderait. Parce qu'il prendrait le risque de mourir, ou parce qu'il placerait la liberté au-dessus de la vie, le peuple ferait la démonstration immédiate, et par la force, de sa maturité politique. C'est pourquoi l'intervention des peuples libres dans les guerres civiles étrangères peut être elle-même immédiate : parce que la guerre civile ne fait qu'actualiser la liberté qui se réalise nécessairement dans l'histoire, et parce que, de ce fait, les peuples ont les uns pour les autres une sympathie, l'intervention est non seulement légitime, mais souhaitable.

\section{«A Few words on Non-Intervention»: la guerre civile comme « test »}

\section{De «Vindication » à «A Few Words » : la différence temporelle}

De l'article de 1849 à celui de 1859, il semble y avoir un renversement total de perspectives: tandis que «Vindication» défendait l'intervention dans les guerres civiles en vertu d'une «sympathie» entre les peuples libéraux, voire d'une identité du «libéralisme » en lutte, «A Few Words » se prononcent clairement contre l'intervention militaire. Mill en énonce ainsi la raison :

[...] On ne peut atteindre que rarement une quelconque assurance que l'intervention, même si elle est réussie, se fasse au profit des gens eux-mêmes. La seule chose qui vaut réellement pour preuve qu'un peuple est devenu capable de vivre sous des institutions populaires [the only test possessing any real value, of a people's having become fit for popular institutions] est que le peuple, ou une partie suffisamment importante pour prendre l'ascendant dans la lutte, soit prêt à braver l'effort et le danger pour sa libération ${ }^{10}$.

Ces quelques lignes permettent de mesurer tout l'écart qui existe entre l'article de 1849 et celui de 1859. En effet, ce qui les distingue du texte que nous avons cité plus haut, c'est bien la prise en compte de la temporalité de l'intervention et de la guerre civile. Cette prise en compte est perceptible, semble-t-il, en quatre endroits.

C'est en premier lieu l'issue probable de l'intervention qui est interrogée ici, ou encore son avenir. Ce qui devrait être pris en compte dans l'intervention, ce n'est pas simplement la victoire militaire que le peuple en armes, assisté de l'armée d'un autre État, pourrait obtenir sur son 
gouvernement tyrannique, mais c'est également la pérennité, la permanence, de sa liberté nouvellement acquise. Autrement dit, ce que suggère Mill ici, c'est que pour justifier une intervention, il ne serait pas suffisant de renvoyer à une histoire libérale commune, mais il faudrait être en mesure d'anticiper et de montrer que la liberté que l'on offre au peuple en lutte est durable, et qu'il ne sera pas réduit en esclavage «en quelques années ou en quelques mois ». Or, comme il le montre, cette anticipation n'est pas possible au début de la guerre civile.

C'est ensuite la «capacité » politique, ou encore «l'aptitude » à avoir des institutions libres qui est pensée dans sa dimension temporelle. Cette idée «d'aptitude » à la liberté pose des problèmes particuliers, que nous n'aborderons pas ici. On peut néanmoins noter que ce que la formule «est devenu capable » [having become fit] indique, c'est que cette «capacité » ou cette « aptitude » n'est pas une donnée naturelle, qui riverait chaque peuple à un type de gouvernement déterminé, mais qu'elle est susceptible de progrès. Or, ce progrès ne se laisse pas reconnaître immédiatement de l'extérieur, mais il doit être vérifié par une mise à l'épreuve, ou, pour reprendre les termes de Mill, par un « test».

C'est bien la «guerre civile» en son entier qui constitue d'autre part ce test. Or, tandis que dans «Vindication», le simple fait de «prendre les armes » constituait un signe suffisant du «souci » de la liberté, ici c'est la guerre civile prise dans sa continuité et dans sa durée qui permettrait d'appréhender l' «aptitude». Dire que le peuple doit être prêt à «braver l'effort et le danger», c'est dire en effet qu'il doit pouvoir persévérer dans la lutte, en dépit de ses difficultés, et assumer tous les moments qui composent la guerre civile.

Enfin, c'est la liberté elle-même qui est pensée ici comme processus : alors que dans «Vindication », Mill parlait de « liberté » ou de « libéralisme en lutte », c'est bien de "libération » qu'il est question ici. Car la guerre civile n'est pas seulement comprise comme un « test», pour les spectateurs étrangers, de l'aptitude à la liberté d'un peuple autrefois esclave, mais elle est également le moment où le peuple devient libre - ou se libère par luimême.

Ainsi, la conclusion paradoxale à laquelle semble conduire l'argumentation de Mill, consiste à dire que si l'intervention est possible, ce ne serait qu'à la fin de la guerre civile, après que le peuple a fait la démonstration de son «aptitude à la liberté » en se libérant. Autrement dit, pour intervenir, nous devrions attendre que le peuple soit devenu libre, c'est-à-dire qu'il ait réussi à soutenir suffisamment longtemps la lutte pour aimer la liberté.

\section{Guerre civile et amour de la liberté}

Ce qui distingue en effet «Vindication » et «A Few Words », c'est bien l'introduction du concept d' «amour de la liberté ». Le problème historique 
et politique posé par l'échec du printemps des peuples et ayant rendu nécessaire l'introduction de cette idée pourrait se formuler ainsi : comment expliquer que des peuples qui sont allés jusqu'à «prendre les armes » pour se libérer, retombent si facilement dans la tyrannie ? Mill formule ainsi sa réponse :

[Si le peuple] n'aime pas la liberté [love of liberty] au point d'être capable de l'arracher à ses oppresseurs entièrement domestiques, la liberté qui lui est dispensée par d'autres mains n'aura rien de réel, rien de permanent. [...] $\mathrm{Si}$ un peuple a eu le malheur d'être dirigé par un gouvernement sous lequel les sentiments et les vertus nécessaires au maintien de la liberté ne pouvaient se développer, c'est au cours d'un rude combat pour être libre que ces vertus ont la meilleure chance d'éclore. Les hommes s'attachent à ce pour quoi, pendant longtemps, ils ont lutté et fait des sacrifices, car ils apprennent à apprécier ce vers quoi leur pensée s'est beaucoup tournée; et un conflit au cours duquel ceux qui ont été appelés à se dévouer pour leur pays sont nombreux est une école qui leur apprend à donner une valeur plus grande aux intérêts de leur pays qu'au leur ${ }^{11}$.

On peut faire ici trois remarques. Premièrement, si l'«amour de la liberté » semble être ici ce qui donne à la liberté sa consistance, ou son contenu réel, Mill n'explique pas vraiment en quoi il consiste. Ce que Castoriadis écrit sur la stasis, dans un cours de 1985 consacré à Thucydide, peut nous éclairer sur ce point ${ }^{12}$. Montrant que la guerre civile ne suffit pas à faire advenir la démocratie, il écrit : «Le grand nombre, le peuple, n'instaure son pouvoir que quand à la fois il prend conscience de sa force et que naît en lui le désir non seulement de ne pas être opprimé [...] mais de se gouverner soi-même » (p. 269). Il rajoute quelques lignes plus bas : «[...] pour qu'il y ait démocratie, il faut dépasser le simple désir d'être protégé contre l'oppression : il faut que naisse le désir de se gouverner soi-même » (p. 270). Le texte de Castoriadis semble faire parfaitement écho au texte de Mill que nous avons cité plus haut. En effet, l'accent est mis dans les deux cas sur la nécessité, pour l'avènement de la démocratie, d'un désir, ou d'un amour, qui ne serait pas négatif - «être protégé contre l'oppression », ou renverser la tyrannie - mais de part en part positif. Autrement dit, ce que nos deux auteurs semblent montrer, c'est qu'il ne suffit pas de vouloir la liberté pour être libre, mais qu'il faut également désirer l'être par soi-même.

Un autre trait semble autoriser la rencontre entre Mill et Castoriadis. En effet, pour l'un comme pour l'autre, l'avènement de la démocratie n'est pas d'abord fonction d'un rapport de forces qui lui serait favorable, mais bien plutôt d'un désir. Pour Castoriadis, la «prise de conscience de sa force » par le peuple semble strictement contemporaine du désir de se gouverner - on pourrait dire ainsi que c'est seulement à partir du moment où le peuple fait le

11. J. S. Mill, ibid., p. 122.

12. C. Castoriadis, Ce qui fait la Grèce (3). Thucydide, la force et le droit : séminaires 19841985, Paris, Seuil, 2010. 
projet de se libérer par lui-même qu'il découvre sa propre force, et qu'il peut l'actualiser. Dans le texte de Mill que nous venons de citer, la question du rapport de force, ou du nombre des belligérants, semble également secondaire. Mill écrit bien que ceux qui sont appelés à se battre pour la liberté doivent être «nombreux », et il insiste également sur le fait que les «forces» contre lesquelles le peuple doit se battre dans la guerre civile doivent être «domestiques ». Cependant, le nombre et la force apparaissent comme des conditions nécessaires mais non suffisantes, puisqu'elles ne garantissent ni la victoire militaire ni le succès politique: seul un attachement affectif à la liberté autorise la libération véritable, qui est tout autant transformation de soi que victoire sur l'adversaire.

Deuxièmement, c'est la désignation de la guerre civile comme «école » qui doit retenir notre attention. Rappelons que pour Mill, l'éducation du peuple par des élites éclairées est un enjeu majeur du gouvernement populaire. Dans son essai de 1830 sur Tocqueville, Mill montrait déjà que l'égalité des conditions, en se généralisant à l'ensemble des pays dits «civilisés », pouvait donner lieu aussi bien à des gouvernements démocratiques qu'à des gouvernements tyranniques. Mill écrivait en effet : «[...] lorsqu'il ne restera plus d'individu ni de classe capable de présenter séparément un obstacle sérieux à la volonté du gouvernement, alors, à moins que le peuple ne soit apte à gouverner, le monarque sera un autocrate aussi parfait que dans la situation d'égalité qui est celle du despotisme asiatique. Lorsque tous sont égaux, il faut qu'ils soient identiquement libres ou esclaves. » (p. 53). De cet essai de 1830 à «A Few Words », une même intuition est à l'œuvre : les institutions libres reposent sur une «aptitude » à la liberté, elles sont donc précaires et fragiles, et peuvent facilement se renverser en tyrannie. L'éducation du peuple devient alors, dans les deux cas, le seul moyen de le rendre apte à se gouverner lui-même. Dans l'essai de 1830, commentant les analyses de Tocqueville, Mill montrait qu'en Amérique, c'était les institutions municipales qui rendaient possible "l'éducation politique du peuple» (p. 58): la participation politique aux affaires communales rendant ainsi possible «la genèse du patriotisme et de l'esprit public » (p. 60). L'instruction scolaire ne jouait en vérité dans l'apprentissage de la vie démocratique qu'un rôle mineur: c'est plutôt la participation habituelle aux affaires municipales qui permettrait la «diffusion de l'intelligence et des activités intellectuelles [rendant le peuple] capable de contrôler avec fermeté et pertinence le fonctionnement de son gouvernement ou d'exercer quelque pouvoir dans les affaires nationales autrement qu'à travers des poussées d'humeur et comme instrument des mains d'autrui » (p. 58).

La guerre civile, pour autant qu'elle soit «longue » et « rude», jouerait ainsi le rôle des «institutions municipales » en démocratie. Cependant, leur fonction n'est pas tout à fait la même : tandis que la participation aux institutions communales vise à faire comprendre au peuple les règles et les enjeux de la vie démocratique, la guerre civile a pour fin de les lui faire aimer. En outre, tandis que les institutions municipales permettent 
d'entretenir une liberté déjà acquise, la guerre civile est véritablement ce qui la fait émerger: elle transforme un simple projet politique abstrait - se libérer du joug d'un tyran -, en un désir ou en un amour, et par là elle modifie l'être de ceux qui luttent. Ceci ne signifie pas qu'elle soit à chaque fois l'occasion d'une telle libération - mais simplement qu'elle est le moment nécessaire de cette médiation. Intervenir dans la guerre civile, pour aider un peuple en lutte pour sa liberté, ce serait donc l'empêcher de s'approprier cette liberté qui est pourtant son projet.

Troisièmement, comme nous l'avons déjà noté, la guerre civile, pour produire, cette différence politique doit durer : «Les hommes s'attachent à ce pour quoi, pendant longtemps, ils ont lutté et fait des sacrifices [Men become attached to that which they have long fought for and made sacrifices for] », écrit Mill dans ce texte déjà cité. Que l'auteur des "Few Words » ne donne pas de limites a priori à cette durée pourrait s'expliquer aisément : si aucune borne n'est assignable, ou encore si ce temps ne connaît pas de mesure, c'est précisément parce que chaque peuple doit se libérer selon un rythme qui lui est propre. Cependant, quelques lignes plus haut, avant de justifier le principe de non-intervention, Mill évoquait les circonstances dans lesquelles une intervention était souhaitable. Il écrivait alors :

Un cas qui mérite l'attention est celui d'une guerre civile prolongée [ $a$ protracted civil war], au cours de laquelle les parties en conflit sont si équilibrées qu'aucune issue rapide n'est probable; ou bien quand la partie victorieuse ne peut espérer dominer la partie vaincue sinon par des traitements qui répugnent à l'humanité et qui dégradent durablement les conditions d'existence ${ }^{13}$.

On voit donc apparaître ici une tension : d'un côté la longueur de la guerre, libératrice, serait affectée de positivité ; de l'autre, le prolongement de la guerre serait compris négativement comme enlisement. Ainsi, la guerre civile, dans son prolongement, pourrait aussi bien être le temps de la liberté que celui de la violence la plus radicale : puisque seule la cruauté - «des traitements qui répugnent à l'humanité »- pourrait permettre à une guerre civile qui excède son temps de se terminer. Toute la question est alors de savoir comment juger du bon temps de la guerre civile, ou encore comment déterminer quand le temps libérateur peut se renverser en temps de la cruauté. La frontière entre l'intervention et la non-intervention semble par là même mouvante, puisqu'elle repose en dernière instance sur la capacité à anticiper l'issue de la guerre civile, ou encore à déterminer sa durée «probable». De même que le gouvernement libre peut rapidement se renverser en tyrannie, et que la libération dans la guerre civile peut conduire au massacre, de même, la non-intervention apparaît profondément réversible. Tout se passe donc comme si le passage de la non-intervention à celui de l'intervention était toujours possible dans le temps de la guerre civile. 


\section{« The Contest in America » : la guerre civile comme régénération}

\section{Prolongement de la guerre et « montée aux extrêmes »}

La question du prolongement de la guerre civile est également au cœur de l'article que Mill consacre à la guerre de Sécession américaine, en 1862 : «The Contest in America». Ce conflit occupe une place importante dans sa pensée: il le commente abondamment dans sa correspondance, et il est le seul auquel il consacre un développement conséquent dans son Autobiographie ${ }^{14}$. C'est que cette guerre, loin de concerner seulement l'Amérique, constitue pour Mill un moment décisif dans l'histoire mondiale. Il écrit en effet : «Mes sentiments les plus forts étaient engagés dans cette lutte, laquelle, je le sentais depuis le début, était destinée à constituer un tournant, en faveur du bien ou du mal, du cours des affaires humaines pour une durée indéfinie » (p. 266).

Spectateur attentif de cette guerre, Mill s'efforce d'en saisir la nature. Or, une des thèses essentielles de son article consiste à dire que le conflit est voué à se transformer dans le temps. Cette thèse, fondée sur l'observation et sur une connaissance des « lois de la nature humaine», fonde alors chez Mill une attente: celle que la guerre dure «suffisamment longtemps » pour produire une telle transformation. Il écrit en effet :

Mais les partis, dans une guerre civile prolongée [a protracted civil war], finissent presque invariablement par adopter des principes plus extrêmes, pour ne pas dire plus élevés, que ceux avec lesquels ils avaient commencé. Les partis modérés et les amis du compromis sont rapidement laissés en arrière [...]. Sans avoir la moindre prétention à voir davantage dans le futur que les autres, j'ai néanmoins prévu et annoncé depuis le début que si le Sud n'était pas rapidement écrasé [if the South were not promptly put down], le conflit deviendrait distinctement anti-esclavagiste [the contest would become distinctly an anti-slavery one]; et je pense qu'aucune personne, habituée à réfléchir au cours des affaires humaines dans les temps troublés, ne peut s'attendre à autre chose. Ceux qui ont lu, même rapidement, le témoignage le plus valable auquel le public anglais ait accès, en ce qui concerne l'état réel de la situation en Amérique - les lettres du correspondant du Times, M. Russel - doivent avoir observé comment il est arrivé tôt et rapidement à la même conclusion, et avec quelle emphase croissante il la répète maintenant continuellement. Dans un de ces courriers récents, il désigne la fin de l'été prochain comme étant la période à laquelle la guerre, si elle ne s'est pas terminée plus tôt, adoptera un caractère anti-esclavagiste complet [he names the end of the next summer as the period by which, if the war has not sooner terminated, it will have assumed a complete anti-slavery character]. Que le terme de cette guerre arrive si tôt correspond, je le confesse, à mes espoirs les plus optimistes ; mais si M. Russel a raison, que le ciel nous préserve que la guerre cesse plus tôt, car si elle dure jusque là, il est tout à fait possible qu'elle permette la régénération du peuple américain [for

14. J. S. Mill, Autobiography, in Autobiography and Literary Essays, CW, vol. I, p. 1-290. 
if it lasts till then it is quite possible that it will regenerate the American people $]^{15}$.

Ce texte appelle deux remarques. Rappelons premièrement que dès le début de cet article, Mill s'efforce de prouver que le Nord n'avait pas initialement l'intention de s'attaquer à l'institution de l'esclavage, défendue par les États du Sud. Il aurait été question, pour le Nord, non de s'en prendre à cette institution, qui relevait des affaires internes des États du Sud, mais plutôt de lutter contre son extension à de nouveaux territoires ${ }^{16}$. On peut se demander ce qui rend si urgente une telle démonstration. Car, si pour Mill, la lutte contre l'esclavage, n'est au fond qu'une lutte du «bien » contre le «mal », ou encore de la «civilisation » contre la barbarie, alors on ne voit pas ce qui l'empêche de proclamer dès le début le caractère abolitionniste de la lutte menée par les Etats du Nord. L'obstacle théorique ou conceptuel à une telle reconnaissance réside, semble-t-il, dans le principe de nonintervention. En effet, comme Mill le rappelle quelques lignes avant notre texte, la constitution américaine interdit l'interférence du Congrès contre l'esclavage dans les États qui le pratiquent ${ }^{17}$. Dire que le Nord était dès le début abolitionniste, ce serait affirmer qu'il ne reconnaît pas la Constitution et le principe sur lequel elle repose, et par là, reconnaître un droit d'intervention. Tout se passe donc comme si la guerre civile américaine n'était pas seulement considérée par Mill comme mettant aux prises le

15. J. S. Mill, Op. cit., p. 135.

16. L'analyse de Mill apparaît sur ce point assez pertinente. En effet, comme le remarque Farid Ameur dans La Guerre de Sécession, le problème de la genèse de la guerre civile et la question de savoir si l'esclavage a été « l'agent moteur de la désunion », continue de se poser et d'animer les débats entre historiens. Cependant, il apparaît que c'est bien contre l'extension de l'esclavage aux nouveaux territoires de l'Ouest que Lincoln et le parti républicain entendaient d'abord lutter. À propos de la convention républicaine de 1860, Farid Ameur écrit : « [...] il n'entre pas dans les intentions du parti de chercher à abolir l'esclavage dans les États de l'Union où il existe déjà. En revanche, son objectif est de circonscrire le mal en s'opposant farouchement à son extension dans les nouvelles terres, pour y faire triompher le principe du travail libre ». (F. Ameur, La Guerre de Sécession, Paris, PUF, 2004, p. 36).

17. Mill écrit en effet : «Les membres du parti républicain ont pris position sur la loi et sur la Constitution existante de l'Union. Ils ont rejeté le droit d'entreprendre tout ce qu'elle interdit. Celle-ci interdit en effet l'interférence du Congrès fédéral contre l'esclavage dans les États esclavagistes ; mais elle n'interdit pas de l'abolir dans la région de Columbia; et c'est ce qu'ils viennent de faire en votant, comme je l'ai compris, un million de dollars d'indemnités pour les propriétaires d'esclaves de la région [...]. Selon les Républicains, la Constitution n'exige pas non plus qu'ils autorisent l'introduction de l'esclavage dans les territoires qui ne sont pas encore des États. C'est pour empêcher ceci que le parti républicain a été créé et qu'il se bat maintenant, de même que les propriétaires d'esclaves luttent pour l'imposer » (p. 132). C'est à la Constitution de 1787 que Mill pense ici, et en particulier à la section 9 de l'article I, où l'on peut lire: «L'immigration ou l'importation de telles personnes telles que l'un quelconque des États actuellement existants jugera convenable d'admettre ne pourra être prohibée par le Congrès avant l'année mille huit cent huit, mais un impôt ou un droit n'excédant pas 10 dollars par tête pourra être levé sur cette importation ». Notons que dans cette section de la Constitution, qui interdit au Congrès de prohiber la traite des esclaves, le terme d'interférence ou d'intervention n'apparaît pas. 
«bien » et le «mal », mais également le principe de non-intervention et son contraire. Autrement dit, la guerre civile américaine devient un test pour le principe de non-intervention ${ }^{18}$. Cette hypothèse appelle cependant une autre question : en effet, si Mill tient à montrer qu'à l'origine, la lutte menée par le Nord contre le Sud n'avait pas pour fin l'abolition de l'esclavage, la transformation progressive de la guerre civile en guerre contre l'esclavage, ne fait-elle précisément pas basculer la guerre en une guerre d'intervention ? Notons que l'auteur des «Few Words », passe ce problème sous silence. Il semble cependant qu'il y ait là encore une manifestation de la réversibilité de la non-intervention, dont on pourra voir un autre exemple plus loin.

Deuxièmement, le prolongement de la guerre est ici compris de manière positive. Il n'est plus question dans ce texte, comme dans «A few words », d'enlisement, ou de durée indéfinie qui ne pourrait être rompue que par la violence. L'expression «protracted civil war », qui apparaissait dans les «Quelques mots » pour désigner les guerres civiles appelant l'intervention, est ici affectée de positivité. En outre, la durée de la guerre n'est pas ce qui permet ici à « l'amour de la liberté » d'apparaitre - elle est plutôt la cause d'une moralisation de la guerre, moralisation qui se comprend comme un retour aux principes qui fondent la démocratie américaine.

Les termes utilisés ici par Mill peuvent rappeler la manière dont Clausewitz, dans De la Guerre, décrit le passage "aux extrêmes » de la guerre, par l'action réciproque des adversaires ${ }^{19}$. Cette « ascension », décrite dans le chapitre I du Livre I, repose sur "l'action réciproque des forces et des volontés aux prises, chacune cherchant à imposer sa loi à l'autre $»^{20}$. Analysant conceptuellement la guerre, en l'isolant «de ses origines et de ses fins », Clausewitz montrait d'abord qu'elle conduisait logiquement à la plus grande violence, avant de donner à voir comment la guerre réelle était toujours soumise à une volonté politique, et ne correspondait donc jamais à son concept.

Le point de vue de Mill est cependant tout autre : il n'analyse pas le concept de la guerre civile, et ses propos se fondent, comme il l'écrit dans notre texte, sur une réflexion effectuée à partir d'un matériau empirique, «les affaires humaines dans les temps troublés ». Dans son Autobiographie, il précisera d'ailleurs que l'idée selon laquelle le prolongement de la guerre civile en modifierait les raisons ou les motifs repose sur «les lois de la nature humaine et l'expérience des révolutions ».

En outre, ce qu'il cherche à montrer, ce n'est pas que la guerre civile aurait tendance à devenir de plus en plus violente, ou encore que ses différents partisans tendraient progressivement à vouloir anéantir leur adversaire, mais plutôt que leurs principes deviennent de plus en plus clairs :

18. Ce texte met en outre en lumière l'articulation entre non-intervention militaire et nonintervention économique - mais nous n'aborderons pas cette question ici.

19. C. Von Clausewitz, De la Guerre, trad. D. Naville, Paris, Éd. de Minuit, 1955, p. 53-54.

20. R. Aron, Penser la guerre, Clausewitz, I, L’âge européen, Paris, Gallimard, 1976, p. 110. 
la guerre civile adopte peu à peu un «caractère anti-esclavagiste bien distinct », ou «complet », écrit Mill. La guerre civile révélerait donc peu à peu à ses protagonistes leurs propres motivations, leur permettant ainsi de les assumer complètement. Que la démocratie américaine soit profondément, ou essentiellement, anti-esclavagiste, va pour Mill de soi : mais c'est cette évidence que la guerre civile rappelle, quand elle se prolonge, aux partisans $\mathrm{du}$ Nord, leur permettant ainsi de faire retour à leur origine, ou de se régénérer.

\section{De la non-intervention à la croisade : le «dilemme» de John Stuart Mill}

Ainsi, il ne saurait être question, pour John Stuart Mill, d'intervenir dans cette guerre civile : même si on peut souhaiter qu'elle se termine vite, il faut en même temps espérer qu'elle ne s'achève pas trop tôt. L'opposition à l'intervention semble à première vue plus radicale que dans «A Few Words », puisque Mill n'envisage pas la situation où la guerre civile excéderait son temps propre. Cependant, dans les dernières pages du texte, il semble adopter une tout autre perspective. Il écrit en effet :

Comme il est nécessaire de se préparer à toutes les possibilités, envisageons-en une autre. Imaginons la pire issue possible de cette guerre [...]. Supposons que le Nord s'abaisse à reconnaître la nouvelle confédération selon ses propres termes, en lui laissant la moitié des territoires, qu'elle soit reconnue par l'Europe, et prenne sa place comme un membre admis de la communauté des nations $[\ldots]$. Devrions-nous regarder cette armée victorieuse autorisée à propager sa foi nationale à coups de fusil à travers le Mexique et l'Amérique centrale ? Devrions-nous accepter de voir le feu et l'épée portés à Cuba et Porto Rico, Haïti et le Libéria conquis et ramenés à l'esclavage ? [...] Le temps pourrait venir où le pouvoir barbare et barbarisant, dont notre soutien moral a favorisé l'existence, rendra nécessaire une croisade générale de l'Europe civilisée, pour anéantir le mal qu'elle a permis, et que nous avons concouru à faire émerger, au milieu de notre civilisation [The time might come when the barbarous and barbarizing Power, which we by our moral support had helped into existence, would require a general crusade of civilized Europe, to extinguish the mischief which it had allowed, and we had aided, to rise up in the midst of our civilization $]^{21}$.

Ainsi, si l'intervention n'est pas possible pendant la guerre civile, parce qu'il faut lui laisser l'occasion de révéler les principes moraux qui la sous-tendent, elle devient nécessaire, le cas échéant, après le conflit. On peut donc dire, d'abord, que la non-intervention chez Mill n'équivaut pas à la neutralité : c'est précisément parce qu'il veut voir les principes défendus par le Nord triompher qu'il s'oppose à l'intervention pendant la guerre civile - mais c'est pour la même raison qu'il se prononce en faveur d'une intervention si les partisans de l'esclavagisme triomphaient. Il faut noter,

21. J. S. Mill, ibid., p. 140-141. 
ensuite, que Mill ne parle pas en fait d' «intervention », dans notre texte, mais de «croisade». Il assume donc ici une idée qu'il refusait dans De la Liberté, puisqu'il y critiquait l'idée d'une "civilisade », c'est-à-dire d'une «croisade civilisatrice» contre les Mormons. La question de savoir si l'intervention est une forme moderne de la croisade, si elle en est la continuation ou la reprise, occupe une place importante dans les débats philosophiques sur l'«interférence» au XIX ${ }^{\mathrm{e}}$ siècle $^{22}$. Nous ne nous attarderons pas sur ce point; mais nous ferons plutôt remarquer que si Mill parle ici de «croisade» plutôt que d' «intervention», ce n'est pas simplement pour produire un effet rhétorique, mais parce que la guerre qu'il préconise excède la simple intervention.

La guerre civile américaine, on l'a dit, a pour Mill une importance cruciale: elle engage en effet la possibilité de la régénération de la démocratie américaine, mais aussi et surtout l'avenir de la civilisation. Les esclavagistes ne sont pas simplement les « ennemis » des États du Nord, ils sont également des «ennemis du genre humain» (p. 163) : la guerre civile n'est donc pas simplement locale, mais elle est une "guerre de principe » qui met aux prises la justice et l'injustice. Dans les dernières lignes de son article, Mill écrit en effet : «Aussi longtemps que la justice et que l'injustice n'ont pas terminé leur combat sans cesse renouvelé pour l'ascendance dans les affaires humaines, les êtres humains doivent vouloir, quand cela est nécessaire, se battre pour l'une contre l'autre. » (p. 167). Ainsi, alors que les guerres civiles évoquées dans «Vindication» ou «A Few Words» avaient pour enjeu la libération régionale des peuples, celui de la guerre civile américaine n'est autre que le triomphe de la «justice» au sein de la civilisation. Parce que la civilisation est une, et qu'elle ne peut tolérer ce qui, en son sein, va à rebours du progrès, on peut dire qu'il n'existe plus vraiment de guerre civile, au sens d'un conflit qui se déroulerait à l'intérieur des frontières d'un Etat: la guerre civile a tendance, pour reprendre les termes de Carl Schmitt à devenir «mondiale», et l'intervention, par là même, généralisée ${ }^{23}$.

22. Sur ce point, nous nous permettons de renvoyer à un article que nous avons publié récemment : " "Civilisade" et intervention chez les peuples barbares selon John Stuart Mill », in Implications philosophiques, Janvier 2011 (http://www.implications-philosophiques.org).

23. Comme le rappelle Céline Jouin dans une préface, l'expression « guerre civile mondiale » [Weltbürgerkrieg] apparaît pour la première fois dans un essai de Schmitt publié en 1943, Changement de structure du droit international. Ce qui est visé par cette expression, explique-t-elle, ce sont «les institutions internationales qui disloquent les États-nations et ouvrent une ère sanglante de guerres post-nationales menées au nom de la paix, de la justice et de la démocratie » (C. Schmitt, La Guerre civile mondiale, essais 1943-1978, trad.C. Jouin, Paris, Ère, 2007, p. 13). Il faudrait comparer avec les textes de Mill, la genèse de la "guerre civile mondiale » et de l'interventionnisme, proposée par Carl Schmitt dans l'essai de 1943, et approfondie dans Le Nomos de la terre en 1955. Il nous semble en effet que cette comparaison pourrait mettre en lumière les limites de la reconstruction historique proposée par Carl Schmitt, au sens où le «dilemme entre neutralité et interventionnisme », contemporain, selon lui, de l'émergence de la puissance américaine à la fin du $\mathrm{XIX}^{\mathrm{e}}$ siècle, nous paraît être déjà présent dans les textes de John Stuart Mill. 


\section{Conclusion}

Quels sont donc les trois modèles du rapport qu'un État libre devrait entretenir avec un peuple qui se libère dans une guerre civile ?

Le premier serait celui de la participation immédiate au conflit : parce que les guerres civiles ne seraient que l'actualisation d'un «libéralisme » vers lequel les peuples s'achemineraient nécessairement, il serait légitime qu'ils s'assistent mutuellement dans le conflit. Toute guerre deviendrait alors guerre de la liberté contre la tyrannie - la question d'identifier « qui lutte et contre qui » devenant ici secondaire.

Le second serait celui de la suspension de l'action, et de la nonintervention : la guerre civile étant le temps dans lequel un peuple autrefois esclave peut apprendre à être libre et à l'être de façon durable, il s'agirait de ne pas contrevenir à ce mouvement. Chaque guerre civile constituerait ainsi un moment propre, absolument singulier dans lequel, à chaque fois, la possibilité de la liberté se rejouerait.

Enfin, le troisième modèle serait celui de l'attente et de l'intervention différée : ce que le spectateur d'une guerre civile attendrait, en effet, ce serait que le conflit conduise les partisans à préciser leurs positions et à les rendre morales. Il y aurait ainsi un devenir moral de la guerre civile ${ }^{24}$, et c'est la raison pour laquelle il serait nécessaire de lui laisser suivre son cours. Cependant, ce serait précisément parce qu'elle aurait bientôt pour enjeu la victoire d'un principe moral sur l'autre, qu'elle pourrait appeler, en cas de victoire du «mal» ou de «l'injustice», l'intervention militaire. La civilisation à laquelle appartiendraient les États libres ne pourrait en effet admettre aucune régression, et quand les guerres civiles régénératrices échoueraient, elle devrait se lancer dans des croisades contre ce qui la menace.

Il faudrait se demander ce qui conduit Mill à adopter progressivement ces trois modèles - ou encore pourquoi il pense d'abord la guerre civile sous l'horizon du mouvement historique du libéralisme, pour ensuite la penser dans sa durée propre comme temps même de l'émancipation, et pour la concevoir enfin comme enjeu moral pour la civilisation. Notons que ces trois modèles semblent encore jouer dans les débats qui continuent à agiter

24. Michael Walzer, dans Guerres justes et injustes, parle d'une «réalité morale de la guerre »: loin que la morale constitue un ensemble de normes, édictées par des théoriciens extérieurs au conflit, le langage moral serait au contraire celui que les participants au conflit utilisent pour décrire leurs propres expériences. Il écrit ainsi : «Répétés au cours du temps, nos arguments et nos jugements ont fini par façonner la réalité morale de la guerre c'est-à-dire, toutes ces expériences que décrit le langage de la morale ou au cours desquelles son usage se révèle indispensable. »(p. 66-67) Pour Mill, cependant, la guerre civile n'a pas d'emblée une véritable dimension morale : la «réalité morale» de la guerre civile, pour l'auteur de «The Contest in America», ne consisterait donc pas dans le fait que ses protagonistes utiliseraient toujours le langage de la justification, pour évaluer les responsabilités de chacun, mais plutôt dans la manière dont ils seraient progressivement amenés à saisir les enjeux essentiellement moraux de la lutte. 
aujourd'hui l'opinion internationale au sujet de l'intervention militaire. Ce qui apparaît en tout cas ici, c'est la nécessité, lorsqu'on veut penser la non-intervention de façon conséquente, de concevoir la liberté non comme un contenu politique pré-donné, constituant un modèle à reproduire, mais comme un processus réellement autonome, ou encore comme une «création », que la guerre civile pourrait faire émerger. 